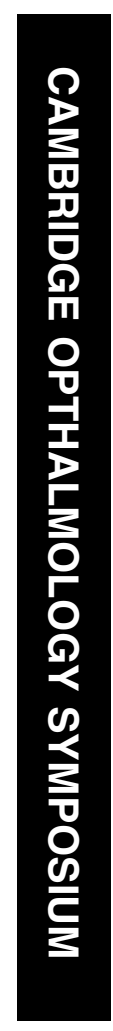

\section{Descemet membrane endothelial keratoplasty (DMEK) for Fuchs endothelial dystrophy: review of the first 50 consecutive cases}

Abstract

Purpose: To evaluate the clinical outcome and complications of Descemet membrane endothelial keratoplasty (DMEK), using Descemet-stripping endothelial keratoplasty (DSEK) as a back-up procedure, in the management of Fuchs endothelial dystrophy. Design: Non-randomised prospective clinical study.

${ }^{1}$ Netherlands Institute for Innovative Ocular Surgery, Rotterdam, The Netherlands

${ }^{2}$ Melles Cornea Clinic Rotterdam, Rotterdam, The Netherlands

${ }^{3}$ Amnitrans Eye Bank Rotterdam, Rotterdam, The Netherlands

Correspondence: GRJ Melles, Netherlands Institute for Innovative Ocular Surgery, Rotterdam,

The Netherlands

Tel: + 31102974444 ; Fax: + 3110297 4440;

E-mail: melles@

niioc.nl

Received: 24 November 2008

Accepted: 25 November 2008

Published online: 30 January 2009

Funding: None
Methods: The first fifty consecutive eyes that underwent DMEK, that is, transplantation of an isolated donor Descemet membrane carrying its endothelium, for Fuchs endothelial dystrophy were evaluated. In all eyes, the best-corrected visual acuity (BCVA) as well as the endothelial cell density (ECD) was measured before and at 6 months after surgery, as clinical outcome parameters. Results: Ten patients required a secondary DSEK for failed DMEK. In the remaining 40 DMEK eyes, $95 \%$ had a BCVA of $\geqslant 20 / 40$ $(\geqslant 0.5)$ and $75 \% \geqslant 20 / 25(\geqslant 0.8)$ at 6 months after surgery. ECD averaged $2618( \pm 201)$ cells $/ \mathrm{mm}^{2}$ before, and $1876( \pm 522)$ cells $/ \mathrm{mm}^{2}$ at 6 months after surgery $(n=35)$. When the outcomes of DMEK and secondary DSEK procedures were combined, 94\% reached a BCVA of $\geqslant 20 / 40$ $(\geqslant 0.5)$ and $66 \% \geqslant 20 / 25(\geqslant 0.8)(n=47)$, and ECD averaged $2623( \pm 193)$ cells $/ \mathrm{mm}^{2}$ before, and $1815( \pm 578)$ cells $/ \mathrm{mm}^{2}$ at 6 months after surgery $(n=43)$. Conclusion: With DSEK as a back-up procedure, DMEK may provide relatively quick and complete visual rehabilitation in a majority of patients operated on for Fuchs
L Ham ${ }^{1,2}$, I Dapena ${ }^{1,2}$, C van Luijk ${ }^{1,2}$, J van der Wees ${ }^{1,3}$ and GRJ Melles s,2,3 $^{1,2}$ endothelial dystrophy. Endothelial cell survival may be similar to earlier types of (lamellar) keratoplasty. Early graft detachment was the main complication in this first series of DMEK surgeries for Fuchs endothelial dystrophy.

Eye (2009) 23, 1990-1998; doi:10.1038/eye.2008.393; published online 30 January 2009

Keywords: Descemet membrane endothelial keratoplasty; posterior lamellar keratoplasty; corneal transplantation; Descemet membrane; surgical technique; Fuchs endothelial dystrophy

Introduction

Since 1998, we introduced various techniques for endothelial keratoplasty, that is, the replacement of the corneal endothelium without corneal surface incisions or corneal sutures, as an alternative to penetrating keratoplasty (PK) in the management of corneal endothelial disorders. ${ }^{1-6}$ These techniques have been popularised in the United States as 'deep lamellar endothelial keratoplasty' (DLEK), ${ }^{7,8}$ and (femtosecond) Descemet stripping (automated) endothelial keratoplasty (DSEK/DSAEK/FS-DSEK) ${ }^{9-12}$ In 1998, we also described a technique for selective transplantation of Descemet membrane through a self-sealing $3.5 \mathrm{~mm}$ clear corneal incision, ${ }^{13,14}$ tentatively named Descemet membrane endothelial keratoplasty (DMEK). ${ }^{15-18}$

As PK has been the gold standard for keratoplasty for almost a century, ${ }^{19}$ an 
alternative technique should bring major advantages in clinical outcome, reducing complications as well as technical feasibility, to get accepted and to change a longstanding consensus in ophthalmology. The early forms of endothelial keratoplasty, that is, DLEK and DSEK/

DSAEK, may have shown that the visual outcome could compete with PK, whereas the most frequent long-term complications were minimised. ${ }^{1-11}$ Endothelial keratoplasty shows little induced astigmatism because the recipient corneal surface is not compromised; suturerelated problems are eliminated as endothelial keratoplasty requires no (corneal) sutures; and wound healing-related complications are rare, because instead of a circular corneal wound, the procedure can be performed through a self-sealing limbal or scleral tunnel incision.

In the current prospective study, we evaluated what clinical outcome and/or complications may be anticipated in patients with Fuchs endothelial dystrophy managed by DMEK, with DSEK as a back-up procedure in the event of DMEK graft failure.

\section{Materials and methods}

Fifty consecutive eyes of 46 patients suffering from Fuchs endothelial dystrophy underwent a DMEK procedure, as described earlier. ${ }^{14,15}$ Twenty-three patients were men and 27 women, ranging from 41 to 88 years of age (Table 1). All patients signed an IRB-approved informed consent.

From donor globes obtained less than $36 \mathrm{~h}$ postmortem, corneo-scleral buttons were excised and stored by organ culture in modified minimum essential medium at $31^{\circ} \mathrm{C} .{ }^{17,18}$ After 1 week of culture, endothelial cell morphology and viability were evaluated and the corneo-scleral buttons were mounted endothelial side up on a custom made holder with a suction cup. Descemet's membrane (DM) was stripped from the posterior stroma, so that a $9.0-10.0 \mathrm{~mm}$ diameter flap of posterior DM with its endothelial monolayer was obtained..$^{21}$ Owing to the elastic properties of the membrane, a 'Descemet-roll' formed spontaneously, with the endothelium at the outer side. Each Descemet-roll was then stored in organ culture medium until the time of transplantation.

In recipient eyes, a $9.0 \mathrm{~mm}$ diameter epithelial mark was made to outline the area of DM excision. A $3.0 \mathrm{~mm}$ tunnel incision was made just within the limbus, entering the anterior chamber just at the mark. With an inversed Sinskey hook (D.O.R.C. International, Zuidland, The Netherlands), a circular portion of DM was scored and stripped from the posterior stroma, so that a $9.0 \mathrm{~mm}$ diameter 'descemetorhexis' was created, and the central portion of DM was removed from the eye.,
The donor Descemet-roll was stained with a $0.06 \%$ trypan blue solution (VisionBlue ${ }^{\mathrm{TM}}$ D.O.R.C.

International), and sucked into a custom made injector (Hippocratech, Rotterdam, The Netherlands), to transfer the tissue from the culture medium vial to the anterior chamber. ${ }^{15,17,18}$ Using the injector, the donor Descemetroll was inserted into the anterior chamber and the graft was oriented endothelial side down (donor DM facing recipient posterior stroma) by careful, indirect manipulation of the tissue with air and fluid. While maintaining the anterior chamber with fluid and air, the graft was gently spread out over the iris. Then, an air bubble was injected underneath the donor DM to position the tissue onto the recipient posterior stroma. ${ }^{15,17,18}$ The anterior chamber was completely filled with air for 30-60 min followed by an air-liquid exchange to pressurise the eye. Each surgical procedure was recorded on DVD (Pioneer DVR-RT601H-S, Tokyo, Japan).

In the eye bank, donor endothelial cell density and viability were evaluated in vitro with an inverted light microscope (Axiovert 40, Zeiss, Göttingen, Germany), and digital photographs were made (PixeLINK PL-A662, Zeiss, Göttingen, Germany). ${ }^{17}$ In patient eyes, the endothelium was photographed and evaluated in vivo using a Topcon SP3000p non-contact autofocus specular microscope (Topcon Corp, Tokyo, Japan). Images of the central corneal window were analysed and manually corrected and three measurements of endothelial cell density were averaged.

\section{Results}

For evaluating the outcome of our study on a first series of 50 consecutive eyes with Fuchs endothelial dystrophy managed by DMEK, two different approaches seem relevant. From a patient's perspective, the overall visual recovery is of interest, to answer the question: 'If DMEK is performed for Fuchs endothelial dystrophy, with DSEK as a back-up procedure in the event of graft failure, what percentage of eyes reaches 20/40 (0.5) or 20/25 (0.8)?' From a scientific perspective, it may be more appropriate to differentiate between the outcome of various endothelial keratoplasty procedures, that is, to evaluate only the successful DMEK cases, to answer the question: 'What visual potential holds DMEK?' Hence, both approaches are displayed below.

\section{Patient's perspective: 6 months visual outcome of corneas transplanted with DMEK, using DSEK as a back-up procedure in the event of DMEK graft failure}

Of the 50 eyes included in our study, 40 eyes had a successful primary DMEK (Figure 1). Ten eyes required a 


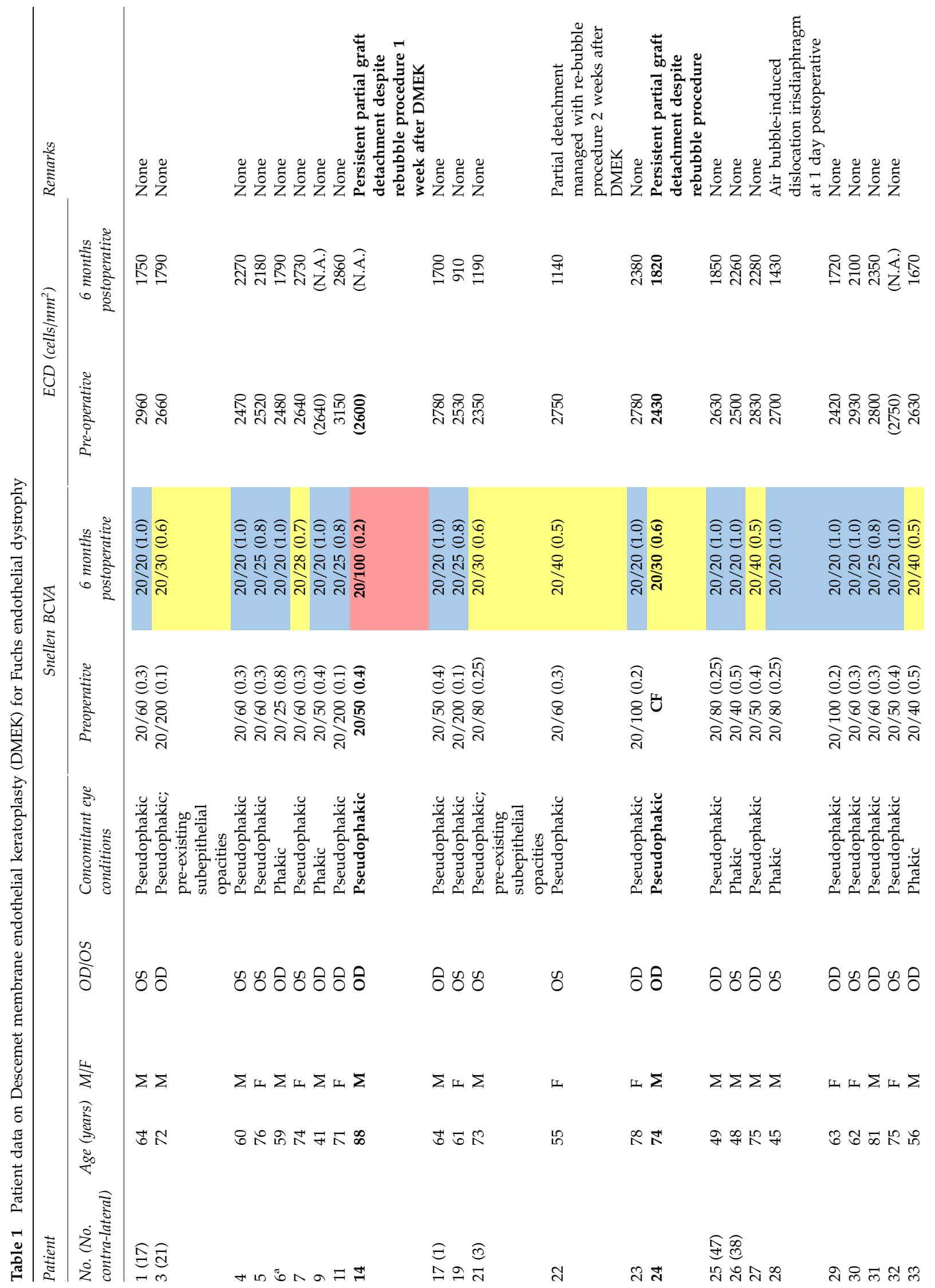




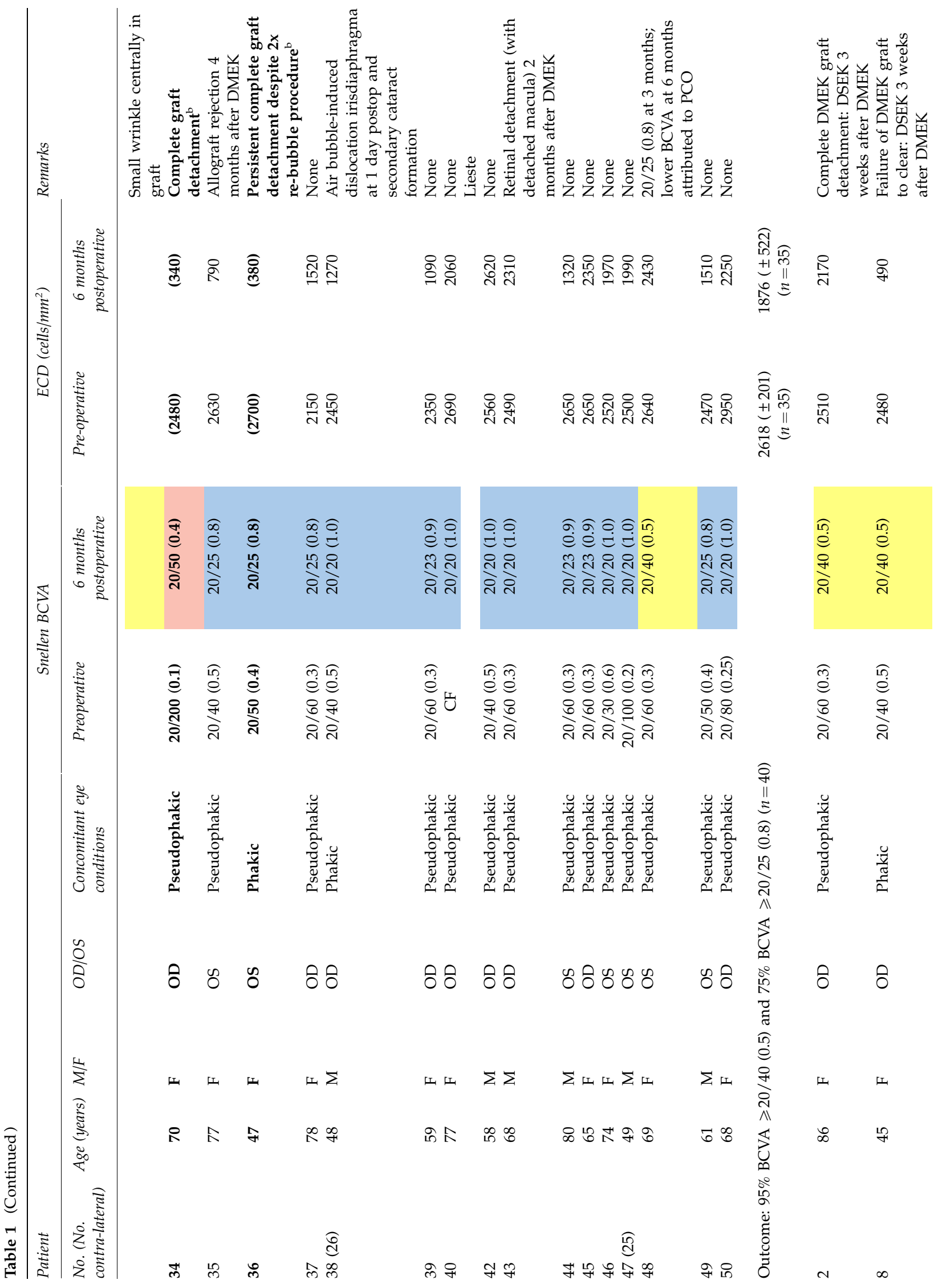




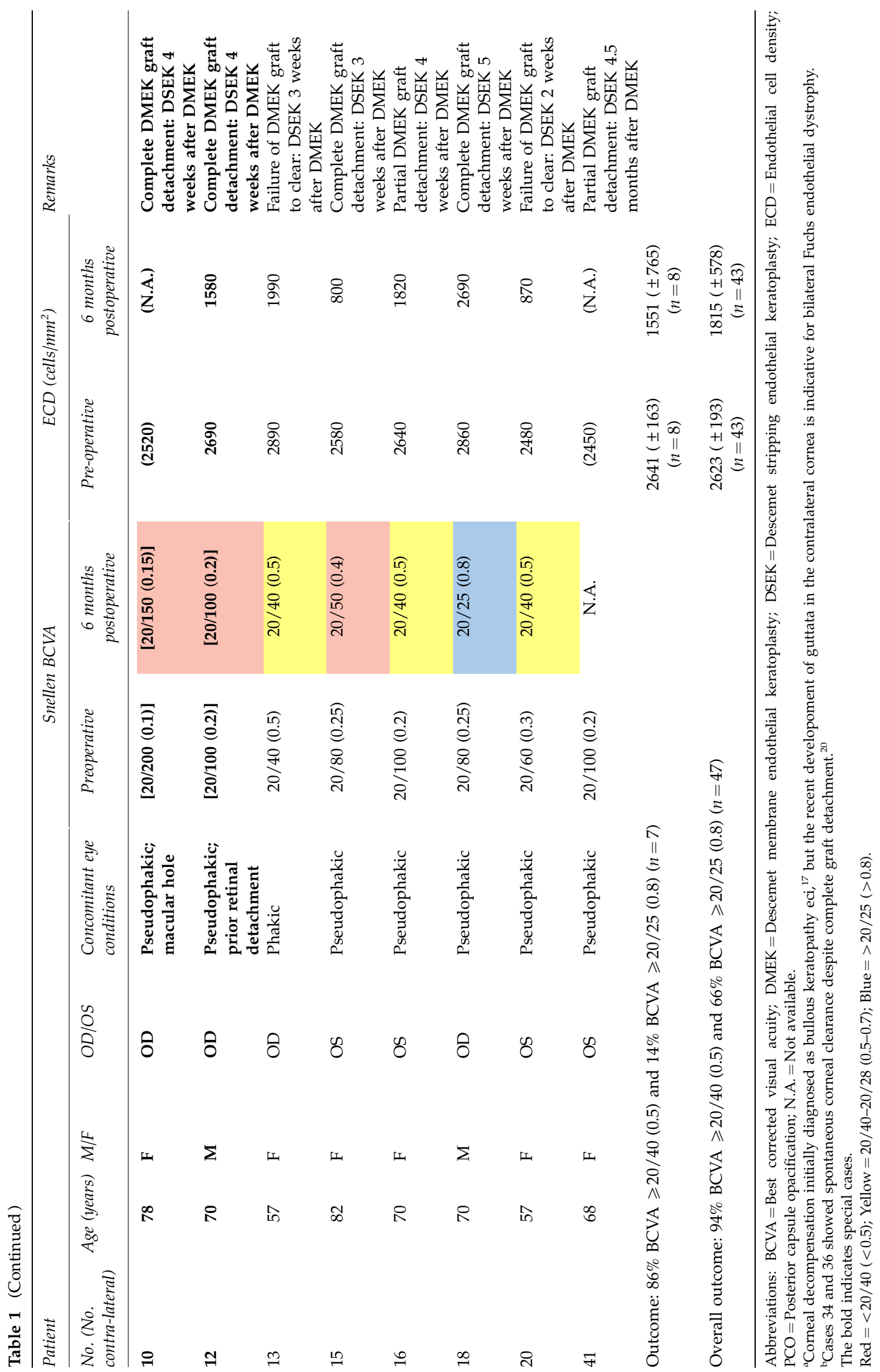



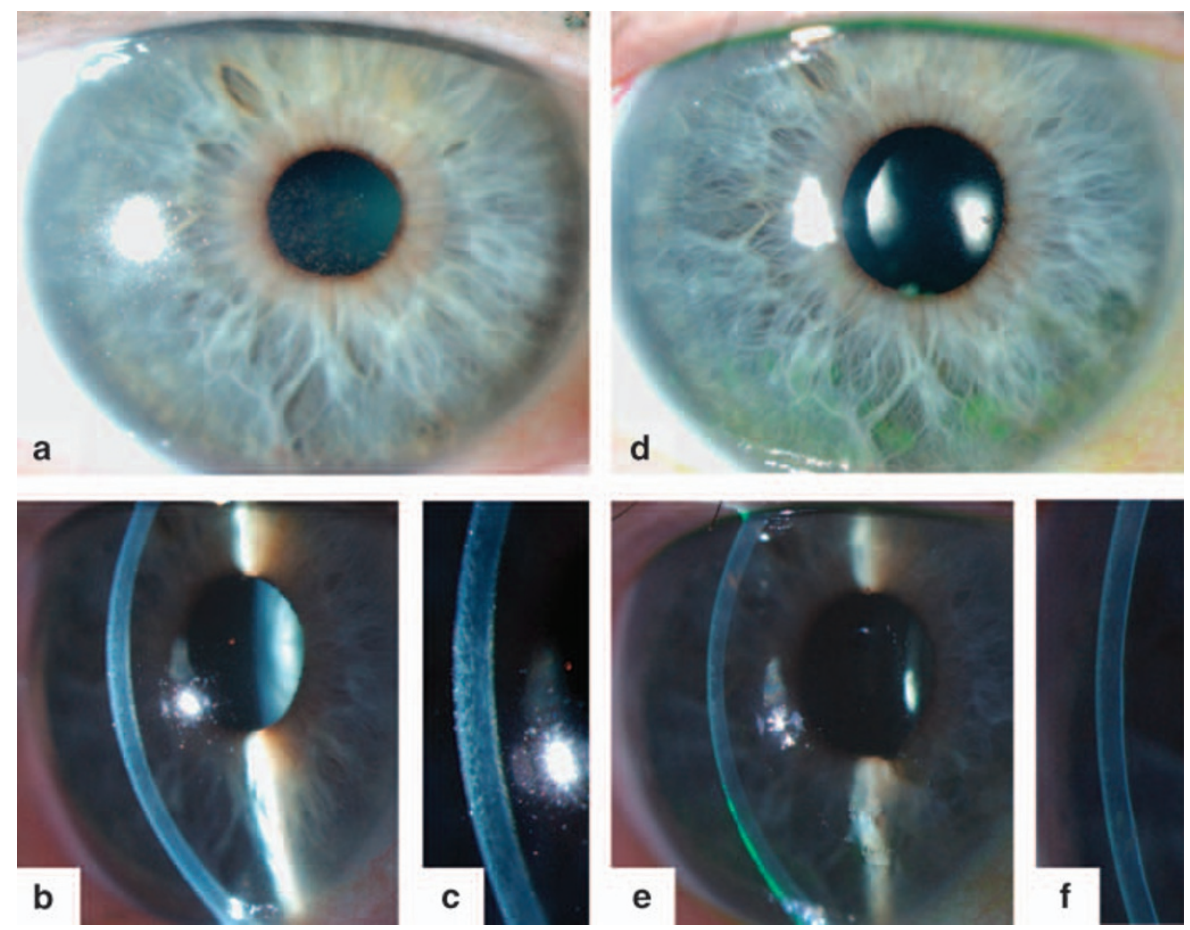

Figure 1 Slit-lamp photographs of an eye (case 50) before $(a-c)$ and at 3 months $(\mathrm{d}-\mathrm{f})$ after Descemet membrane endothelial keratoplasty (DMEK) for Fuchs endothelial dystrophy. Note the near anatomical restoration of the transplanted cornea as well as its clarity after DMEK.
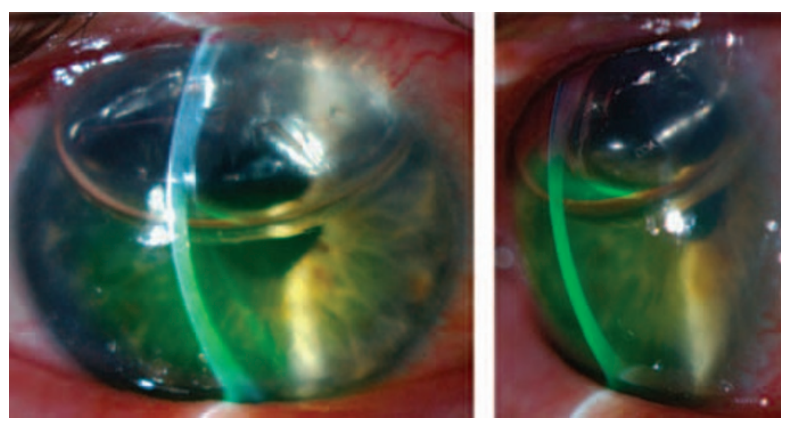

Figure 2 Slit-lamp photograph of an eye (case 47) 1 day after Descemet membrane endothelial keratoplasty (DMEK). Note the relative clarity of the transplanted cornea. At 1 day postoperative, the BCVA was $0.6(20 / 30)$, despite the presence of the $50 \%$ air-fill of the anterior chamber.

secondary DSEK because of complete or partial DMEK graft detachment (cases 2, 10, 12, 15, 16, 18, and 41; Table 1) or failure of the transplanted cornea to clear (cases 8, 13, and 20; Table 1). Of the 50 eyes, 47 eyes (40 DMEK and 7 secondary DSEK) had good visual potential and were available for examination (Cases 10, 12, and 41 were excluded from analysis; Table 1). Of the latter eyes, $94 \%(44 / 47)$ achieved a best corrected visual acuity (BCVA) of $20 / 40$ or better $(\geqslant 0.5)$ and $66 \%(31 / 47)$ reached $20 / 25$ or better $(\geqslant 0.8)$ at 6 months after surgery
(Table 1). Three of these 47 eyes did not reach the 20/40 (0.5), which was attributed to partial graft detachment in case 14 and graft detachment in case 34 , and case 15 had a low visual acuity despite uneventful secondary DSEK surgery and complete clearance of the transplanted cornea (Table 1).

\section{Scientific perspective: Visual outcome of transplanted corneas with DMEK grafts in situ at 6 months}

In 40 eyes the DMEK graft was in situ at 6 months. In these eyes, 95\% had a BCVA of $\geqslant 20 / 40(\geqslant 0.5)$ and $75 \%$ reached $\geqslant 20 / 25(\geqslant 0.8)$ at 6 months after surgery. Interestingly, all of the eyes with a functional DMEK graft obtained a BCVA of $20 / 40$ or better $(\geqslant 0.5)$. In the 10 DMEK eyes that did not reach $20 / 25$ or better $(\geqslant 0.8)$ (cases 3, 7, 14, 21, 22, 24, 27, 33, 34, and 48; Table 1), the reduced BCVA could not be explained in three eyes $(10 \%)$ (cases 7, 22, and 27). In the remaining seven patients, a visual recovery limited to 0.5 to 0.7 (20/40 to $20 / 28$ ) was attributed to secondary subepithelial stromal scarring due to long-standing dystrophic changes in one patient with bilateral DMEK surgery (cases 3 and 21; Table 1); partial graft detachment just below the corneal centre (cases 14 and 24; Table 1); the presence of a small wrinkle in the central graft area (case 33; Table 1); partial corneal clearance despite complete graft detachment 
(case 34; Table 1); and the development of posterior capsule opacification (Case 48; Table 1).

\section{DMEK endothelial cell density}

Of the 50 eyes included in our study, pre- and postoperative endothelial cell density (ECD) measurements were available in 43 eyes (35 DMEK and 8 secondary DSEK). The overall donor ECD averaged 2623 ( \pm 193 ) cells $/ \mathrm{mm}^{2}$ before, and $1815( \pm 578)$ cells $/ \mathrm{mm}^{2}$ at 6 months after surgery (Table 1). Of the 40 DMEK eyes, pre- and postoperative ECD measurements were available in 35 eyes, and donor ECD averaged 2618 $( \pm 201)$ cells $/ \mathrm{mm}^{2}$ before, and $1876( \pm 522)$ cells $/ \mathrm{mm}^{2}$ after surgery (Table 1 ). Of the 10 eyes with a secondary DSEK procedure, ECD measurements were available in eight eyes. Donor ECD averaged $2641( \pm 163)$ cells $/ \mathrm{mm}^{2}$ before, and $1551( \pm 765)$ cells $/ \mathrm{mm}^{2}$ after surgery (Table 1). No significant difference in preoperative as well as postoperative ECD was found among the groups $(P>0.1)$. The drop in ECD between preoperative and postoperative ECD was significant in all groups $(P<0.05)$.

\section{Complications}

Seven eyes developed a graft detachment, characterised by the presence of a free-floating Descemet-roll in the anterior chamber (cases 2, 10, 12, 15, 18, 34, and 36; Table 1) and five eyes a partial detachment (cases 14, 16, 22, 24, and 41; Table 1). Seven of these twelve (partial) detachments were managed by a secondary DSEK procedure (cases 2, 10, 12, 15, 16, 18, and 41; Table 1), and one case with a partial detachment (case 22; Table 1) had a successful re-bubbling procedure. In two cases with a partial detachment, the clinical outcome was satisfactory to the patient (cases 14 and 24; Table 1). Two cases (cases 34 and 36; Table 1) showed spontaneous corneal clearance despite graft detachment, with visual recoveries to $20 / 50(0.4)$ and $20 / 25(0.8) .{ }^{20}$ In three eyes (cases 8, 13, and 20; Table 1) that did not show corneal clearance within the first weeks after surgery, a secondary DSEK procedure was performed.

Two cases (cases 28 and 38, Table 1) showed imminent angle-closure glaucoma due to entrapment of residual air behind the iris, with forward dislocation of the iris diaphragm at the first operative day. Although the air bubble could be repositioned towards the anterior chamber by pupillary dilatation, both cases developed a mild anterior subcapsular cataract similar to 'Glaukomflecken' for which a phaco-emulsification was performed at 6 months postoperatively in case 38. One eye (case 35; Table 1) showed an allograft rejection at 4 months after DMEK, and a retinal detachment occurred in a (anatomically) high myopic eye at 2 months after surgery in case 43 (Table 1).

\section{Discussion}

Our study shows that in a first series of 50 consecutive Fuchs endothelial dystrophy patients that underwent a primary DMEK and if indicated a secondary DSEK procedure, the far majority of eyes obtained a Snellen visual acuity of $20 / 40$ or better $(\geqslant 0.5)$, the threshold for a driving license in various countries. When the three eyes with low visual potential or unavailability were excluded from analysis, over $90 \%$ of cases achieved a BCVA of $20 / 40$ or better $(\geqslant 0.5)$ and two-third reached $20 / 25$ or better $(\geqslant 0.8)$ at 6 months after surgery (Table 1$)$. When secondary DSEK eyes were excluded from analysis, that is, when only eyes with a DMEK graft in situ were considered, $\frac{3}{4}$ of cases showed (nearly) complete visual recovery to $20 / 25$ or better $(\geqslant 0.8)$. In the latter eyes, the rate of visual rehabilitation was found to be unprecedented: one-third of eyes obtained $20 / 40$ or better $(\geqslant 0.5)$ within 1 week (Figure 2$)$, and virtually all eyes reached their maximal visual potential at 1-3 months. $^{18}$

The overall visual recovery for the whole group as well as that obtained specifically with DMEK, may compare favourably to PK and the early endothelial keratoplasty techniques, like DLEK and DSEK/DSAEK. To the best of our knowledge, there is not a single report in the scientific ophthalmic literature describing a visual outcome in PK even approaching that after DMEK. On average, historic cohort studies on PK for Fuchs endothelial dystrophy report a visual outcome of 20/40 or better at 1 year in only $40-50 \%$ of patients. ${ }^{22}$ These reports may be flattered by contact lens fitting, that commonly meets little compliance with elderly people in the long term. Studies on DSEK/DSAEK generally report a better visual outcome than with PK, with visual acuities up to 20/40 (0.5) in a majority of cases, with only small percentages reaching $20 / 25$ or better $(\geqslant 0.8) .{ }^{10,11,19,23}$ Both in PK and DSEK/DSAEK, a final visual acuity is usually achieved at 6-12 months, whereas most DMEK patients reach their maximal visual potential at $1-3$ months. ${ }^{18}$

When comparing postoperative endothelial cell densities, a second major clinical outcome parameter in keratoplasty surgery, DMEK outcomes may be roughly similar to those after PK or DSEK/DSAEK. Our study shows an initial decline in donor cell density of approximately $30 \%$ at 6 months, mimicking the pre- to postoperative cell loss after other types of keratoplasty. ${ }^{12,24-26}$ Longer term endothelial cell counts may be required to better evaluate how the decline in cell density after DMEK compares to PK and DSEK/DSAEK. As DMEK graft diameters $(9.0-10.0 \mathrm{~mm})$ exceed those in 
PK (7.0-8.0 mm) and DSEK/DSAEK (8.0-9.0 mm), more endothelium is transplanted, thus longer graft survival might theoretically be expected in DMEK.

In the best of hands, PK is often complicated by longterm complications like high and/or irregular astigmatism, suture-related problems and/or late wound disruption. ${ }^{16}$ Although endothelial keratoplasty techniques were designed to minimise these problems, a detailed analysis may be warranted regarding the incidence and severity of complications after DMEK, to evaluate how the latter technique compares to PK. As in DSEK/DSAEK, graft detachment initially stood out as the major drawback of performing DMEK ${ }^{10,17}$ In the current study, (partial) graft detachment in $25 \%(12 / 50)$ of eyes, consistently within the first week after surgery, was also found to be the main complication after DMEK (Table 1). Nine of these detachments were clustered within the first 25 eyes (that is, a $36 \%$ detachment rate), whereas only three detachments occurred in the last 25 eyes (that is, a $12 \%$ detachment rate). Although possibly just due to a learning curve, detachments may also have been reduced by two changes in surgical and eye banking techniques. First, we extended the duration of a complete air-fill of the anterior chamber at termination of the surgery from 30 to $45-60 \mathrm{~min}$. Second, we eliminated the use of plastic vials during the entire tissue processing, for electrostatic charges may somehow relate to changes in surface tension of the relative thin, isolated Desemet graft.

The clinical course of the detachments varied. Five complete detachments were managed with a secondary DSEK procedure (cases 2, 10, 12, 15, and 18), whereas two cases (cases 34 and 36) showed spontaneous corneal clearance despite graft detachment, with visual recovery up to $20 / 25(0.8) .{ }^{20}$ Of the five partial detachments, one case (case 22) had a successful re-bubbling procedure, two were managed by secondary DSEK procedures (cases 16 and 41), and the clinical outcome was satisfactory to the patient in two cases (cases 14 and 24). In three cases (cases 8,13 , and 20; Table 1), the transplanted cornea failed to clear within the first weeks after surgery, despite complete DMEK graft adherence. As all grafts showed acceptable ECDs when examined after explantation in the eye bank, in retrospect the secondary DSEK procedure may have been conducted prematurely. Some Descemet grafts may show no apparent clearance for 6-8 weeks. Case 17 proved a turning point in our clinical management to postpone a secondary surgery if persistent postoperative corneal decompensation is accompanied by complete graft adherence: being scheduled for re-operation, the transplanted cornea showed complete clearance with visual recovery to 1.0 at 2 months after the DMEK surgery.

Other complications following the DMEK surgery included an allograft rejection at 4 months after DMEK (case 35; Table 1), and a retinal detachment in a highly myopic eye at 2 months after surgery (Case 43; Table 1). Both complications seemed isolated events and may therefore be considered coincidental. In two cases (cases 28 and 38; Table 1) an iatrogenic cataract developed due to entrapment of the 50\% air-bubble (left in situ in the anterior chamber at the termination of the surgery) behind the iris, with forward dislocation of the iris diaphragm. Although peripheral iridotomies were routinely made with the Argon-YAG laser prior to DMEK surgery to prevent angle-closure glaucoma, an iridotomy is not effective in preventing glaucoma due to air-bubble dislocation with mechanical displacement of the iris diaphragm. In both cases, displacement of air behind the iris occurred during surgery, so that in cases with peroperative air dislocation, postoperative medication for pupillary dilatation may routinely be prescribed.

Compared to DSEK/DSAEK, a major advantage of DMEK may be that the donor Descemet can be stripped directly from a corneo-scleral rim, without the need for a microkeratome or femtosecond laser. As donor corneal tissue is commonly distributed as corneo-scleral rims by most eye banks throughout the world, the accessibility to endothelial keratoplasty may be far better with DMEK than with DSEK/DSAEK. Even in third world countries or in low volume keratoplasty centres with a limited budget for equipment, DMEK may prove a feasible technique although requiring minimal financial investment.

In conclusion, our study shows that DMEK is a feasible procedure with a relatively short learning curve, although DMEK may require specific training in surgical technique and tissue handling. With regard to visual outcome and endothelial cell density as clinical outcome parameters, the incidence and severity of complications, as well as technical and logistic feasibility, DMEK may soon be preferred over PK and DSEK/DSAEK, in the management of Fuchs endothelial dystrophy.

Financial interest: Dr Melles is a consultant for D.O.R.C./ Dutch Ophthalmic USA. GM and JvdW contributed to the design of the study. LH, ID, CVL, JvdW and GM contributed to the conduct of the study and the data analysis. The study was conducted in compliance with the Institutional Review Board and informed consent requirements, in adherence to the tenets of the Declaration of Helsinki, at the Netherlands Institute for Innovative Ocular Surgery (study registration no. N.05.14).

\section{References}

1 Melles GRJ, Eggink FAGJ, Lander F, Pels E, Rietveld FJR, Beekhuis WH et al. A surgical technique for posterior lamellar keratoplasty. Cornea 1998; 17: 618-626. 
2 Melles GRJ, Lander F, Beekhuis WH, Remeijer L, Binder PS. Posterior lamellar keratoplasty for a case of pseudophakic bullous keratopathy. Am J Ophthalmol 1999; 127: 340-341.

3 Melles GRJ, Lander F, van Dooren BTH, Pels E, Beekhuis WH. Preliminary clinical results of posterior lamellar keratoplasty through a sclerocorneal pocket incision. Ophthalmology 2000; 107: 1850-1857.

4 Melles GRJ, Lander F, Nieuwendaal C. Sutureless, posterior lamellar keratoplasty. Cornea 2002; 21: 325-327.

5 Melles GR, Wijdh RH, Nieuwendaal CP. A technique to excise the descemet membrane from a recipient cornea (descemetorhexis). Cornea 2004; 23: 286-288.

6 Melles GRJ, Kamminga N. Techniques for posterior lamellar keratoplasty through a scleral incision. Ophthalmologe 2003; 100: 689-695.

7 Terry MA, Ousley PJ. Deep lamellar endothelial keratoplasty in the first United States patients: early clinical results. Cornea 2001; 20: 239-243.

8 Terry MA, Ousley PJ. Small-incision deep lamellar endothelial keratoplasty (DLEK): six-month results in the first prospective clinical study. Cornea 2005; 24: 59-65.

9 Price Jr FW, Price MO. Descemet's stripping with endothelial keratoplasty in 200 eyes. Early challenges and techniques to enhance donor adherence. J Cataract Refract Surg 2006; 32: 411-418.

10 Price MO, Price FW. Descemet's stripping endothelial keratoplasty. Curr Opin Ophthalmol 2007; 18: 290-294.

11 Gorovoy MS. Descemet-stripping automated endothelial keratoplasty. Cornea 2006; 25: 886-889.

12 Cheng YY, Hendrikse F, Pels E, Wijdh RJ, van Cleynenbreugel H, Eggink CA et al. Preliminary results of femtosecond laser-assisted descemet stripping endothelial keratoplasty. Arch Ophthalmol 2008; 126: 1351-1356.

13 Melles GRJ, Rietveld FJR, Pels E, Beekhuis WH, Binder PS. Transplantation of Descemet's membrane carrying viable endothelium through a small scleral incision. Invest Ophthalmol Vis Sci 1998; 39(suppl): S76, abstract no 343.

14 Melles GRJ, Rietveld FJR. Transplantation of Descemet's membrane carrying viable endothelium through a small scleral incision. Cornea 2002; 21: 415-418.
15 Melles GRJ, Ong TS, Ververs B, van der Wees J. Descemet membrane endothelial keratoplasty (DMEK). Cornea 2006; 25: 987-990.

16 Melles GRJ. Posterior lamellar keratoplasty. DLEK to DSEK to DMEK. (editorial). Cornea 2006; 25: 879-881.

17 Melles GRJ, Ong TS, Ververs B, van der Wees J. Preliminary clinical results of Descemet membrane endothelial keratoplasty (DMEK). Am J Ophthalmol 2008; 145: 222-227.

18 Ham L, Balachandran C, Verschoor CA, van der Wees J, Melles GRJ. Visual rehabilitation rate after isolated Descemet membrane transplantation: Descemet membrane endothelial keratoplasty (DMEK). Arch Ophthalmol 2009; in press.

19 Bahar I, Kaiserman I, McAllum P, Slomovic A, Rootman D. Comparison of posterior lamellar keratoplasty techniques to penetrating keratoplasty. Ophthalmology 2008; 115: 1525-1533.

20 Balachandran C, Ham L, Verschoor MA, Ong TS, van der Wees J, Melles GRJ. Spontaneous corneal clearance despite graft detachment in Descemet membrane endothelial keratoplasty (DMEK). Am J Ophthalmol 2009; in press.

21 Lie JT, Van der Wees J, Melles GRJ. Donor tissue preparation for Descemet membrane endothelial keratoplasty (DMEK). J Cataract Refract Surg 2008; 34: 1578-1583.

22 Williams KA, Muehlberg SM, Lewis RF, Coster DJ. How successful is corneal transplantation? A report from the Australian Corneal Graft Register. Eye 1995; 9: 219-227.

23 Chen ES, Terry MA, Shamie N, Hoar KL, Friend DJ. Descemet-stripping automated endothelial keratoplasty: six-month results in a prospective study of 100 eyes. Cornea 2008; 27: 514-520.

24 van Dooren BTH, Mulder P, Nieuwendaal CP, Beekhuis WH, Melles GRJ. Endothelial cell density after posterior lamellar keratoplasty (Melles technique); 3 years follow-up. Am J Ophthal 2004; 138: 211-217.

25 Busin M, Bhatt PR, Scorcia V. A modified technique for descemet membrane stripping automated endothelial keratoplasty to minimize endothelial cell loss. Arch Ophthalmol 2008; 126: 1133-1137.

26 Chen ES, Terry MA, Shamie N, Phillips PM, Friend DJ, McLeod SD. Descemet-stripping automated endothelial keratoplasty: insertion using a novel 40/60 underfold technique for preservation of donor endothelium. Cornea 2008; 27: 941-943. 